\title{
Investigation on Slot/Pole Number Combinations for PMSM with Concentrated Fractional Slot Winding
}

\author{
Guangqi Liu \\ Graduate University, CAS, Beijing 100049, State Key Laboratory of Robotics, Shenyang Institute of \\ Automation, CAS, Shenyang 110016 \\ Liuchee@sia.cn
}

\begin{abstract}
Keywords: Fractional slot, Concentrated winding, Slot/pole number combination, Cell motor, Dummy motor
\end{abstract}

\begin{abstract}
Five features of slot number-phase table of cell motor are deeply discussed in this paper. Then it is derived that four constraints of cell motor with fractional slot winding and two additional constraints of cell motor with concentrated fractional slot winding. Finally, the equivalent calculation of spread factor of fractional slot motor and integral slot motor is proposed by use of the dummy motor. Because Slot number-phase table is brought in, the method proposed by this paper is convenient to carry out automatic analysis and calculation by computer.
\end{abstract}

\section{Introduction}

As is known to all, concentrated fractional slot winding abbreviated as concentrated winding is the winding whose slots per pole per phase is fractional number and the winding pitch y equals to 1 , which means the coil is just wraped arround single tooth of the stator. Therefore, there is no overlapping in the winding overhang and no need to set the phase insulation. As a resualt, the length of coils is shorten, which can reduce the amount of copper to make the resistance smaller and get higher efficiency and power density [3,4]. For instance, as the motor of Sanyo air-conditioning compressor adopts concentrated winding in substitution for integral slot winding, its weight is reduced by $15 \%$, the copper cost by $40 \%$, the copper power loss by $30 \%$, and the running efficiency is improved by $6 \%[4]$. Obviously, concentrated winding is conducive to energy conservation, material saving, motor miniaturization and weight reduction. Generally, concentrated winding is commonly used in low-power, low speed and direct drive applications. In these applications, the motor is expected to have small radial size, light weight and high output torque. So torque motor of flat structure is commonly adopted. Due to the small axial size and aspect ratio, it is difficult to weaken torque ripple by use of oblique slot in stator and pole in $\operatorname{rotor}^{[1]}$. Also, it is difficult to make the motor have integral slots in the small radial size from processing point of view. Fortunately, it is enough to reduce the torque ripple to a very small level only by use of fractional slot without oblique slot or pole ${ }^{[2]}$. Meanwhile, it is very important to shorten the length of winding overhang in the motor of small aspect ratio because of its notable end effect. And selection of slot/pole number combinations is the key step for torque PMSM design. So this paper mainly studies on the slot/pole number combinations features of torque PMSM with concentrated winding.

First, this paper deeply discussed five features of slot number-phase table of cell motor. And then, the equivalent calculation of spread factor of fractional slot motor and integral slot motor was proposed by use of dummy motor. At last, feasible slot/pole number combinations were discussed and summarized into tables.

\section{Slot Number-Phase Table of Cell Motor}

If $\mathrm{t}$ is the greatest common divisor of $\mathrm{Z}$ and $\mathrm{p}$, then we have $\mathrm{Z}=\mathrm{Z}_{0} \mathrm{t}, \mathrm{p}=\mathrm{p}_{0} \mathrm{t}$ and slots per pole per phase is

$$
q=\frac{Z}{2 m p}=\frac{Z_{0}}{2 m p_{0}}
$$

Here we call the motor as cell motor, which is of slots $Z_{0}$, poles $p_{0}$ and phases $\mathrm{m}$. So the original motor is formed by $\mathrm{t}$ cell motors. If we expand the slot-vector star graph[5] into a horizontal line and cast away vector arrowhead. So each circumference becomes a horizontal line 
and each vector becomes a slot number whose position in the horizontal line represents the phase of the corresponding vector. To avoid overlap, any two different slots with the same phase should be written in different levels of line but at the same vertical line. Also, the negative slot number which represents the coils passed through the negative current should be considered. Meanwhile, the positive slot number and its negative counterpart should have the interval of $\pi$. At last we can get a table called slot number-phase table [5], shown as table 1. Now we assume the table has Q columns and $\mathrm{R}$ rows, then each row represents $2 \pi$ electrical angle. And there are 5 features of slot number-phase table of cell motor.

The table must have even columns. The positive slots and its corresponding negative one are fixed $\pi$ electrical angle apart from each other in the table, thus it requires electrical angle $\pi$ should be divided by $2 \pi / Q$, i.e. $Q / 2$ must be an integer. Therefore, the column number of the slot number-phase table must be an even number. So we have $\mathrm{Q}=Z_{0}$ when $Z_{0}$ is even and $\mathrm{Q}=2 Z_{0}$ when $Z_{0}$ is odd.

Grid angle is $2 \pi / Q$. Each grid in the table represents $2 \pi / Q$ electrical angle in the slot numberphase table

$$
\alpha_{X}=\frac{2 \pi}{Q}=\frac{2 \pi p_{0}}{X Z_{0}}=\frac{1}{X} \alpha
$$

where, $\alpha=2 \pi p_{0} / Z_{0}$ is the slot pitch angle of cell motor. Specifically, $\alpha_{X}=2 \pi / Z_{0}, X=p_{0}$ when $Z_{0}$ is even and $\alpha_{X}=\pi / Z_{0}, X=2 p_{0}$ when $Z_{0}$ is odd.

The table has $2 p_{0}$ rows. When $Z_{0}$ is even, we can know that the number of grids in one row is $Z_{0}$ and two adjacent slot numbers will apart from each other by $X=p_{0}$ grids in the table. So $Z_{0}$ slots totally need $Z_{0} p_{0}$ grids, which means there needs to be $Z_{0} p_{0} / Q=p_{0}$ rows to accommodate positive slots and another $p_{0}$ more rows to accommodate negative ones; Similarly, when $Z_{0}$ is odd, the number of grids in one row is $2 Z_{0}$ and two adjacent slot numbers will apart from each other by $X=2 p_{0}$ grids in the table. So $Z_{0}$ slots totally need $2 Z_{0} p_{0}$ grids, which mean there needs to be $2 Z_{0} p_{0} / Q=p_{0}$ rows to accommodate positive slots and another $p_{0}$ more rows to accommodate negative ones. So, slot number-phase table must have $2 p_{0}$ rows regardless of the parity of $Z_{0}$.

Different slots can not be filled into the same grid and slots of the same sign will not be in the same column. If we number the grids in the row wise, the number of the grid in the first row and first column will be 1 . So the number of grid in the rth row and cth column will be (r-1) $\mathrm{Q}+\mathrm{c}$. Therefore, the number of the ith slot will be $(i-1) X+1$ in the table, and the column is

$$
c_{i}=\bmod \left[\frac{(i-1) X+1}{Q}\right]
$$

where mod[] takes the remainder of the fraction in square brackets. So we can know the co-column condition of two different but the same sign slots is that they have the same remainder calculated by (3).

$$
\frac{(i-1) X+1}{Q}-\frac{(j-1) X+1}{Q}=k Q, k=1,2 \ldots, \mathrm{p}_{0}-1
$$

If $Z_{0}$ is odd, (4) can be rewritten as

$$
\frac{p_{0}(i-j)}{Z_{0}}=2 k Z_{0}, 1 \leq k \leq p_{0}-1, i-j \leq Z_{0}-1
$$

So we have,

$$
i-j=\frac{2 k Z_{0}^{2}}{p_{0}} \leq Z_{0}-1
$$


If $Z_{0}$ is even

$$
\frac{p_{0}(i-j)}{Z_{0}}=k Z_{0}, 1 \leq k \leq p_{0}-1, i-j \leq Z_{0}-1
$$

i.e. $i-j=\frac{k Z_{0}^{2}}{p_{0}} \leq Z_{0}-1$

when $Z_{0}$ and $p_{0}$ don't have common divisor, we can prove that i-j can not be an integer, which means $i$ and $j$ are not in the same column.

Supposing that $Z_{0}$ is odd and $2 k / p_{0}$ is integer, so i-j will also be an integer, but $i-j>Z_{0}-1$ because of the discriminant $Z_{0}^{2}-Z_{0}+1>0$; Supposing $2 k / p_{0}$ is a fraction, so $i-j$ will also be a fraction because $Z_{0}$ and $p_{0}$ don't have common divisor. Therefore, we can ensure that any two different slots will not be in the same column when $Z_{0}$ is odd. Similarly, this conclusion is also correct when $Z_{0}$ is even by the same analysis method.

Either single slot or two slots with opposite sign is filled into each column. From feature 1), we know Q must be even. So the parity of the column of slot $i$ is determined by the parity of the grid number $(i-1) X+1$. If $Z_{0}$ is odd, then $X$ equals to $2 p_{0}$ and its grid number is $2 p_{0}(i-1)+1$ which must be an odd number. Because any two different slots with the same sign are not in the same column, so $Z_{0}$ slots can be filled into $Z_{0}$ odd columns of slot number-phase table. Then the negative slots can be filled into even columns of the table because the interval between positive and corresponding negative slots is $Z_{0}$ which is odd. So there is only one positive or negative slot filled into each column when $Z_{0}$ is odd; If $Z_{0}$ is even, then $X$ equals to $p_{0}$ which must be an odd number known from property 4$)$ in section 2 . So the parity of grid number $p_{0}(i-1)+1$ is determined by the parity of $i$. Therefore, $Z_{0}$ slots will be corresponding with $Z_{0}$ columns of the table. Then the negative slots can be filled into another $p_{0}$ more rows of the table and the interval between positive and corresponding negative slots is $Z_{0} / 2$. So each column has two slots with opposite sign when $Z_{0}$ is even. As a result, either single slot or two slots with opposite sign is filled into each column of the table.

For example, if $\mathrm{m}=3, \mathrm{Z}_{0}=9$ and $\mathrm{P}_{0}=4$, we can select $\mathrm{X}=2 \mathrm{P}_{0}=8$ and $Q=X Z / p=18$. So each column of slot number-phase table shown as table 1 has only one slot (positive or negative). Another example is $\mathrm{m}=3, \mathrm{Z}_{0}=18$ and $\mathrm{P}_{0}=5$. Because $\mathrm{Z}_{0}$ is even, so $\mathrm{X}=\mathrm{P}_{0}=5$. And we can get $\mathrm{Q}=18$. As described above, each column has two slots with opposite sign, shown as table 2.

Table 1 The slot number-phase table of cell motor with $m=3, Z=9, p=4$

\begin{tabular}{|l|l|l|l|l|l|l|l|l|l|l|l|l|l|l|l|l|l|}
\hline 1 & & & & & & & & 2 & & & & & & & & 3 & \\
\hline & & & & & & 4 & & & & & & & & 5 & & & \\
\hline & & & & 6 & & & & & & & & 7 & & & & & \\
\hline & & 8 & & & & & & & & 9 & & & & & & & \\
\hline & -9 & & & & & & & & -1 & & & & & & & & -2 \\
\hline & & & & & & & -3 & & & & & & & & -4 & & \\
\hline & & & & & -5 & & & & & & & & -6 & & & & \\
\hline & & & -7 & & & & & & & & -8 & & & & & & \\
\hline
\end{tabular}

The restrictions of $Z_{0} / p_{0}$ Combination for Cell Motor with Concentrated Fractional-slot winding

The $\mathrm{Z}_{0} / \mathrm{p}_{0}$ combination for cell motor with fractional-slot winding should satisfy the following conditions $[3,4]$.

1) Slots per pole per phase should be a fraction, i.e. $q \neq$ integer ; 
2) There should not be common divisor between $Z_{0}$ and $p_{0}$;

3) Slots of cell motor $Z_{0}$ should be integral times of $m$ in order to ensure $m$ phase windings are symmetrical and there is no common divisor between $\mathrm{p}_{0}$ and $\mathrm{m}$.

4) If $Z_{0}$ is even, then $p_{0}$ must be odd.

Table 2 The slot number-phase table of cell motor with $m=3, Z=18, p=5$

\begin{tabular}{|c|c|c|c|c|c|c|c|c|c|c|c|c|c|c|c|c|c|}
\hline 1 & & & & & 2 & & & & & 3 & & & & & 4 & & \\
\hline & & 5 & & & & & 6 & & & & & 7 & & & & & 8 \\
\hline & & & & 9 & & & & & 10 & & & & & 11 & & & \\
\hline & 12 & & & & & 13 & & & & & 14 & & & & & 15 & \\
\hline & & & 16 & & & & & 17 & & & & & 18 & & & & \\
\hline & & & & -18 & & & & & -1 & & & & & -2 & & & \\
\hline & -3 & & & & & -4 & & & & & -5 & & & & & -6 & \\
\hline & & & -7 & & & & & -8 & & & & & -9 & & & & \\
\hline-10 & & & & & -11 & & & & & -12 & & & & & -13 & & \\
\hline & & -14 & & & & & -15 & & & & & -16 & & & & & -17 \\
\hline
\end{tabular}

The condition 2) and 3) also be satisfied when $p_{0}=1$ as a special case. Let y represents coil pitch, then the electrical angle of the coil pithc is

$$
\alpha_{y}=y \alpha=y X \alpha_{X}
$$

and let $\beta=\pi-\alpha_{y}=\pi\left(1-y \frac{2 p_{0}}{Z_{0}}\right)$

$\beta$ is called fractional pitch angle if $2 p_{0} y / Z_{0}<1$; The winding is called full pitch winding if $\beta=0$ and $2 p_{0} y / Z_{0}=1$, which can not be true in fractional windings because of condition 2 ); $-\beta$ is called fractional pitch angle if $2 p_{0} y / Z_{0}>1$. As to two-layer winding, if the winding satisfys above 4 conditions and its winding pitch equals to 1 , then it is called concentrated fractional-slot winding(concentrated winding for abbreviate). Then we can know about concentrated windings that $\alpha_{y}=\alpha$, and $\beta=\pi-\alpha=\pi\left(1-2 p_{0} / Z_{0}\right)$. According to feature 4$)$ in section 1 , the circumference will be equally divided by $Z_{0}$ slots, which means the phase is $2 \pi / Z_{0}$ for each division and electrical angle $\pi$ is corresponding with $Z_{0} / 2$ slots. So when $Z_{0}$ is even, we can set

$$
\beta= \pm N \frac{2 \pi}{Z_{0}}, \mathrm{~N}=1,2 \ldots
$$

According to (10) and (11), we can get

$$
Z_{0}=2 p_{0} \pm 2 N, \mathrm{~N}=1,2,3 \ldots
$$

If $Z_{0}$ is odd, we can set

$$
\beta= \pm 0.5 N \frac{2 \pi}{Z_{0}}, \mathrm{~N}=1,3,5 \ldots
$$

And according to (10) and (13), we can get

$$
Z_{0}=2 p_{0} \pm N, \mathrm{~N}=1,3,5 \ldots
$$

where the "+” represents fractional pitch windings and “-” represents long pitch windings. Then we can get the fifth restriction from (12) and (14).

5) The restrictions of slot/pole number combination for concentrated windings.

$$
\left\{\begin{array}{c}
Z_{0}=2 p_{0} \pm N, N=1,2,3 \ldots \\
\beta=N \pi / Z_{0}=\pi\left(1-2 p_{0} / Z_{0}\right)
\end{array}\right.
$$


So the parity of $Z_{0}$ is the same as that of $\mathrm{N}$ which always equals to 1 or 2 when there are fewer slots. In order to ensure the pitch factor is not less than 0.866 , the absolute value of $\beta$ can't be more than $\pi / 3$. According to (15), we can get

6) pitch factor $k_{y} \geq 0.866$, i.e.

$$
\left\{\begin{array}{l}
-\frac{\pi}{3} \leq \beta \leq \frac{\pi}{3} \\
\frac{Z_{0}}{3} \leq p_{0} \leq \frac{2 Z_{0}}{3} \\
Z_{0} \geq 3 N \\
\frac{3}{4 m} \leq q \leq \frac{3}{2 m}
\end{array}\right.
$$

Among above 6 restrictions, 1) 4)are the restrictions for forming the fractional windings and 5) 6) are the additional restrictions for further forming concentrated windings from the fraction one. Now conventional $\mathrm{Z}_{0} / \mathrm{p}_{0}$ number combinations for 3-phase concentrated windings are listed in Table 3 .

\section{Winding Factor Calculation for Concentrated Windings}

The winding factor $k_{w}$ consist of slot factor $k_{s}$, pitch factor $k_{y}$ and spread factor $k_{q}$. Here, we only disccuss $k_{y}$ and $k_{q}$ because $k_{s}$ is independent with geometric size and spatial distribution of windings. Specifically the pitch factor is defined as

$$
k_{y}=\cos \frac{\beta}{2}
$$

and the spread factor of windings is defined as $k_{q}=\frac{\text { Vector Sum of magnetomotive force or EMF }}{\text { Algebraic Sum of magnetomotive force or EMF }}$.

As to regular $60^{\circ}$ or $120^{\circ}$ phase spread windings with integral slots, the spread factor can be calculated as follows:

$$
k_{q}=\frac{\sin \frac{q \alpha}{2}}{q \sin \frac{\alpha}{2}}
$$

According to the analysis of slot number-phase table in section 1, we can easily conclude that the spread factor only depends on the distribution of the columns of the grids selected by the winding instead of the slot number. When some conditions are satisfied, regular $60^{\circ}$ or $120^{\circ}$ phase spread windings with fractional slot can be equivalent to the two poles $(\mathrm{p}=1)$ dummy motor with integral slots in the sense of calculating the spread factor according to (18). The conditions are as follows

1) The same number of phase $m$

2) The same $Q$, which means the slots of dummy motor is the same as or twice that of cell motor with fractional slot.

3) In the same column, the Slots of dummy motor is the same as or twice that of cell motor with fractional slot.

If $Z_{0}$ is odd, the dummy motor is also a motor with fractional slot. For example, the slots per pole per phase is $3 / 2$, when $m=3, Z=9$ and $p=1$; If $Z_{0}$ is even, the dummy motor is a motor with integral slots For example, the slots per pole per phase is 3 , when $m=3, Z=18$ and $p=1$. According to the three conditions listed above, the difference of the pair of poles $p$ would not make spread factor 
different. So the motor shown in table 1 is equivalent to the motor shown in table 4 , and the motor shown in Table 2 to Table 5 in the sense of spread factor. When $Z_{0}$ is odd, the dummy motor with fractional slot can be further equivalent to dummy motor with integral slots whose slots will be $2 Z_{0}$, pairs of poles $p_{0}=1$ and number of phase $m=3$. For example, the motor shown in table 4 can be considered as the dummy motor shown in Table 5 when we calculate the spread factor of the motor with $\mathrm{q}=3 / 2$.

Table 4 The slot number-phase table of dummy motor with $m=3, Z=9, p=1$

\begin{tabular}{|l|l|l|l|l|l|l|l|l|l|l|l|l|l|l|l|l|l|}
\hline 1 & & 2 & & 3 & & 4 & & 5 & & 6 & & 7 & & 8 & & 9 & \\
\hline & -6 & & -7 & & -8 & & -9 & & -1 & & -2 & & -3 & & -4 & & -5 \\
\hline
\end{tabular}

Table 5 The slot number-phase table of dummy motor with $\mathrm{m}=3, \mathrm{Z}=18, \mathrm{p}=1$

\begin{tabular}{|c|c|c|c|c|c|c|c|c|c|c|c|c|c|c|c|c|c|}
\hline 1 & 2 & 3 & 4 & 5 & 6 & 7 & 8 & 9 & 10 & 11 & 12 & 13 & 14 & 15 & 16 & 17 & 18 \\
\hline-10 & -11 & -12 & -13 & -14 & -15 & -16 & -17 & -18 & -1 & -2 & -3 & -4 & -5 & -6 & -7 & -8 & -9 \\
\hline
\end{tabular}

According to the restrictions proposed in section 2 and the conditions in this section, we can get the commonly used $Z_{0}=2 p_{0} \pm 1, Z_{0}=2 p_{0} \pm 2$ slot/pole number combinations of 3-phase motor with concentrated fractional slot and their spread factors shown in table 6 .

\section{Conclusion}

This paper analyzed 5 features of slot number-phase table of fractional slot motor without common divisor between $Z$ and $\mathrm{p}$. And then, the restrictions and table of slot/pole number combination of cell motor with concentrated winding were proposed. Finally, cell motor with fractional slot can be equivalent to the two poles $(\mathrm{p}=1)$ dummy motor with integral slots in the sense of calculating the spread factor according to the analysis of slot number-phase table. It is very convenient to calculate winding factor by use of slot number-phase table instead of star-vector graph, which is conducive to perform automatic winding analysis using computer.

\section{References}

[1] Wang Xudong, $\mathrm{Xu}$ Xiaozhuo, Feng Haichao. Present Study and Prospect of the Cogging Torque Comprehen sive Reducing Methods of PM Motors [J]. Micromotors,2009,42 (12) :64 70.

[2] Chen Yiguang, Pan Yuling, He Xin. Magnetomotive Force in Permanent Magnet Synchronous Machine With Concentrated Fractional-Slot Winding [J]. Transactions of China Electrotechnical Society, 2010,25(10):30 36.

[3] Mo Huicheng. Fractional-solt winding and PM brushless motor[J]. Micromotors, 2007, 40(11): $39-4281$

[4] Tan Jiancheng. Investigation on slot/pole number combinations for 3-phase BLDCM with concentrated windings[J]. Micromotors, 2007, 40(12): 72-77, 86.

[5] Xu Shizhang. Winding Theory of AC Machine [M]. Machinery Industry Press, Beijing, 1985.

[6] Chen Shiyuanm, Huang Shipeng. Winding Theory of AC Machine [M]. China Electric Power Press, Beijing, 2007. 
Table 3 Conventional $Z_{0} / p_{0}$ Number Combinations for 3-phase PMSM with Fractional S1

\begin{tabular}{|c|c|c|c|c|c|c|c|c|c|c|c|c|c|c|c|c|c|c|}
\hline$Z_{0}$ & 3 & & 9 & & 15 & & 21 & & 27 & & 33 & & 39 & & 45 & & 51 & \\
\hline $\mathrm{P}_{0}$ & & 6 & & 12 & & 18 & & 24 & & 30 & & 36 & & 42 & & 48 & & 54 \\
\hline 1 & $3 / 1$ & & & & & & & & & & & & & & & & & \\
\hline 2 & $3 / 2$ & & & & & & & & & & & & & & & & & \\
\hline 4 & & & $9 / 4$ & & & & & & & & & & & & & & & \\
\hline 5 & & & $9 / 5$ & $\begin{array}{c}12 / \\
5\end{array}$ & & & & & & & & & & & & & & \\
\hline 7 & & & & $\begin{array}{c}12 / \\
7\end{array}$ & $\begin{array}{c}15 / \\
7\end{array}$ & $\begin{array}{c}18 / \\
7\end{array}$ & & & & & & & & & & & & \\
\hline 8 & & & & & $\begin{array}{c}15 / \\
8\end{array}$ & & $\begin{array}{c}21 / \\
8\end{array}$ & & & & & & & & & & & \\
\hline 10 & & & & & & & $\begin{array}{c}21 / \\
10\end{array}$ & & $\begin{array}{l}27 / \\
10\end{array}$ & & & & & & & & & \\
\hline 11 & & & & & & $\begin{array}{c}18 / \\
11\end{array}$ & $\begin{array}{c}21 / \\
11\end{array}$ & $\begin{array}{c}24 / \\
11\end{array}$ & $27 /$ & 30/ & & & & & & & & \\
\hline 13 & & & & & & & 21/ & $24 /$ & $27 /$ & 30/ & 33/ & $36 /$ & & & & & & \\
\hline 13 & & & & & & & 13 & 13 & 13 & 13 & 13 & 13 & & & & & & \\
\hline 14 & & & & & & & & & $27 /$ & & $\begin{array}{l}33 / \\
14\end{array}$ & & $\begin{array}{c}39 / \\
14\end{array}$ & & & & & \\
\hline 16 & & & & & & & & & 27/ & & $33 /$ & & $39 /$ & & $45 /$ & & & \\
\hline 10 & & & & & & & & & 16 & & 16 & & 16 & & 16 & & & \\
\hline 17 & & & & & & & & & 27/ & 30/ & 33/ & $36 /$ & 39/ & $42 /$ & $45 /$ & $48 /$ & & \\
\hline & & & & & & & & & 17 & 17 & 17 & 17 & 17 & 17 & 17 & 17 & & \\
\hline 19 & & & & & & & & & & 30/ & 33/ & $36 /$ & $39 /$ & $42 /$ & $45 /$ & $48 /$ & $51 /$ & $54 /$ \\
\hline 19 & & & & & & & & & & 19 & 19 & 19 & 19 & 19 & 19 & 19 & 19 & 19 \\
\hline 20 & & & & & & & & & & & 33/ & & 39/ & & & & $51 /$ & \\
\hline & & & & & & & & & & & 20 & & 20 & & & & 20 & \\
\hline
\end{tabular}

Table 6 Conventional Winding Factors of 3-phase PMSM with Concentrated Slot

\begin{tabular}{|c|c|c|c|c|c|c|c|c|c|c|c|c|c|c|c|c|}
\hline $\begin{array}{c}\mathbf{Z}_{\mathbf{0}} \\
/ \mathbf{P}_{\mathbf{0}} \\
\end{array}$ & \begin{tabular}{|l|}
3 \\
1 \\
\end{tabular} & \begin{tabular}{|l|}
3 \\
12 \\
\end{tabular} & $\begin{array}{l}9 \\
14 \\
\end{array}$ & $\begin{array}{c}9 \\
15\end{array}$ & $\begin{array}{l}12 \\
15 \\
\end{array}$ & $\begin{array}{l}12 \\
17 \\
\end{array}$ & $\begin{array}{l}15 \\
/ 7 \\
\end{array}$ & $\begin{array}{l}15 \\
/ 8 \\
\end{array}$ & $\begin{array}{c}21 \\
/ 10 \\
\end{array}$ & \begin{tabular}{c|}
21 \\
$/ 11$ \\
\end{tabular} & $\begin{array}{c}24 \\
/ 11 \\
\end{array}$ & $\begin{array}{c}24 \\
/ 13 \\
\end{array}$ & $\begin{array}{c}27 \\
/ 13 \\
\end{array}$ & $\begin{array}{c}27 \\
/ 14 \\
\end{array}$ & \begin{tabular}{|c|}
33 \\
$/ 16$ \\
\end{tabular} & $\begin{array}{c}\mathbf{3 3} \\
/ \mathbf{1 7} \\
\end{array}$ \\
\hline $\mathbf{q}$ & $\stackrel{N}{=}$ & $\stackrel{\Xi}{\Xi}$ & $\frac{\infty}{m}$ & $\stackrel{\rho}{m}$ & $\stackrel{\sim}{\sim}$ & $\stackrel{\Sigma}{\lambda}$ & $\frac{\nabla}{n}$ & $\frac{0}{n}$ & 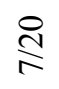 & $\underset{\mathbb{N}}{\mathbb{N}}$ & $\vec{F}$ & $\stackrel{m}{f}$ & $\stackrel{\text { L }}{\text { a }}$ & $\stackrel{\infty}{\stackrel{N}{\alpha}}$ & $\stackrel{\text { હૈ }}{=}$ & $\stackrel{\stackrel{ \pm}{\Xi}}{=}$ \\
\hline $\mathbf{q}^{\prime}$ & $\stackrel{N}{\subseteq}$ & $\stackrel{S}{S}$ & $\frac{N}{m}$ & $\underset{m}{N}$ & $\sim$ & $\sim$ & $\frac{N}{n}$ & $\frac{N}{n}$ & $\stackrel{\curvearrowright}{\curvearrowright}$ & $\stackrel{N}{N}$ & $\nabla$ & $\nabla$ & $\stackrel{N}{a}$ & $\stackrel{N}{a}$ & $\stackrel{N}{\Xi}$ & $\stackrel{N}{\Xi}$ \\
\hline $\mathbf{N}$ & - & - & - & - & $\sim$ & $N$ & - & - & - & - & $N$ & $N$ & - & - & - & - \\
\hline $\boldsymbol{\beta}$ & 8 & 8 & ㄱ & ㄱ & in & లి & $\simeq$ & $\simeq$ & $\begin{array}{l}\tilde{n} \\
\infty\end{array}$ & $\begin{array}{l}\hat{n} \\
\infty\end{array}$ & $\cong$ & $\cong$ & $\begin{array}{l}\hat{b} \\
.\end{array}$ & $\begin{array}{l}\hat{b} \\
0\end{array}$ & $\begin{array}{l}\text { fo } \\
\text { in }\end{array}$ & $\begin{array}{l}\stackrel{n}{f} \\
\dot{n}\end{array}$ \\
\hline $\mathbf{k}_{\mathbf{y}}$ & $\begin{array}{l}0 \\
\stackrel{0}{0} \\
\infty \\
0\end{array}$ & $\begin{array}{l}0 \\
\infty \\
\infty \\
0\end{array}$ & $\begin{array}{l}2 \\
2 \\
0 \\
0\end{array}$ & 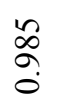 & $\begin{array}{l}8 \\
\circ \\
\circ \\
0\end{array}$ & $\begin{array}{l}\circ \\
\stackrel{0}{\circ} \\
0\end{array}$ & ڤ̆ & ڤ̆ & $\hat{a}$ & $\hat{a}$ & $\bar{\alpha}$ & $\begin{array}{l}\bar{a} \\
\text { o. }\end{array}$ & $\begin{array}{l}\infty \\
\stackrel{\alpha}{\circ}\end{array}$ & 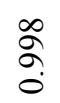 & ڤ. & ळे \\
\hline 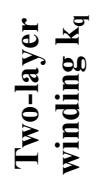 & - & - & $\begin{array}{l}\stackrel{8}{:} \\
\stackrel{0}{0}\end{array}$ & $\begin{array}{l}\stackrel{0}{0} \\
\stackrel{0}{0}\end{array}$ & $\begin{array}{l}0 \\
\stackrel{0}{\circ} \\
0 \\
0\end{array}$ & $\begin{array}{l}8 \\
\stackrel{0}{\circ} \\
0\end{array}$ & $\begin{array}{l}\hat{n} \\
\hat{o}\end{array}$ & ñ. & 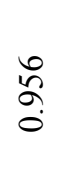 & $\begin{array}{l}\mathscr{0} \\
\stackrel{2}{\circ} \\
o\end{array}$ & $\begin{array}{l}\infty \\
\dddot{o} \\
o\end{array}$ & 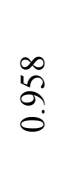 & $\begin{array}{l}n \\
\tilde{o} \\
0\end{array}$ & $\begin{array}{l}n \\
\hat{\sigma} \\
o\end{array}$ & $\begin{array}{l}n \\
\hat{\sigma} \\
o\end{array}$ & $\begin{array}{l}n \\
\hat{o} \\
o\end{array}$ \\
\hline $\mathbf{k}_{\mathrm{w}}$ & $\begin{array}{l}\stackrel{0}{\circ} \\
\stackrel{0}{0} \\
0\end{array}$ & $\begin{array}{l}0 \\
\stackrel{0}{\circ} \\
0 \\
0\end{array}$ & 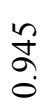 & 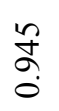 & & $\hat{\tilde{\sigma}}$ & $\begin{array}{l}\bar{n} \\
o \\
0\end{array}$ & ڤ̆ & $\begin{array}{l}\hat{n} \\
\hat{\sigma} \\
o\end{array}$ & $\begin{array}{l}\hat{n} \\
\hat{o} \\
o\end{array}$ & ڤે & ڤे & $\begin{array}{l}\hat{n} \\
\hat{o}\end{array}$ & $\hat{n}$ & \begin{tabular}{l}
\multirow{2}{*}{} \\
$\stackrel{0}{0}$
\end{tabular} & 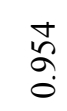 \\
\hline
\end{tabular}

Note: q'represents the slots per pole per phase of the dummy motor 